\title{
A sex-specific linkage map of the white shrimp Penaeus (Litopenaeus) vannamei based on AFLP markers
}

\author{
Franklin Pérez $^{\mathrm{a}, \mathrm{b}, *}$, Constanza Erazo $^{\mathrm{a}}$, Mariuxi Zhinaula ${ }^{\mathrm{a}}$, \\ Filip Volckaert ${ }^{\mathrm{b}}$, Jorge Calderón ${ }^{\mathrm{a}}$ \\ ${ }^{a}$ Fundación CENAIM-ESPOL, Km. 30.5 Via Perimetral, Guayaquil 09014519, Ecuador \\ ${ }^{\mathrm{b}}$ Katholieke Universiteit Leuven, Laboratory of Aquatic Ecology, Ch. de Bériotstraat 32, B-3000 Leuven, Belgium
}

Received 3 June 2004; received in revised form 6 September 2004; accepted 6 September 2004

\begin{abstract}
We report the construction of sex-specific linkage maps for the white shrimp Penaeus (Litopenaeus) vannamei (Penaeidae; Crustacea). Linkage information was generated using amplified fragment length polymorphism (AFLP) markers in a mapping panel consisting of 42 individuals derived from a commercial cross. We used 103 primer combinations that produced 741 segregating bands. From them, 477 segregated in a 1:1 model, 181 in a 3:1 model, 62 fitted both models and 21 fitted neither model. A total of 394 loci with a 1:1 segregation ratio were mapped to unique positions in the male and female maps using a pseudotestcross strategy. A total of 51 and 47 linkage groups were detected for the male and female maps respectively, in comparison to 44 haploid groups expected from the karyotype. The female map covered 2771 Kosambi units (cM) and was $24 \%$ longer than the male map (2116 cM long). The distribution of the markers showed that both maps had low saturation and clustering at short linkage distances. Markers with a distorted segregation were observed as previously reported in other shrimp species. The estimated genomic length indicates that the $P$. vannamei genome has higher recombination rates than closely related species. We demonstrate the feasibility of implementing molecular techniques
\end{abstract}

* Corresponding author. Fundación CENAIM-ESPOL, Km. 30.5 Vía Perimetral, Guayaquil 09014519, Ecuador. Tel.: +5934916118x119; fax: +5934916120.

E-mail address: franklinperez@hotmail.com (F. Pérez). 
at low cost without the need of specialized equipment in a species that is at the initial stages of domestication.

(C) 2004 Published by Elsevier B.V.

Keywords: AFLP; Crustacea; Linkage map; Penaeus (Litopenaeus) vannamei; Parentage testing

\section{Introduction}

Selective breeding and dissection of genetic traits require genetic linkage maps for various purposes such as: characterization of quantitative trait loci (QTL), markerassisted selection (MAS), evolutionary studies by synteny characterization, map-based cloning and contig assembly (Savelkoul et al., 1999; Smith et al., 2002). A common problem to species with either long reproductive cycles or those at an early stage of domestication is the lack of appropriate pedigrees for linkage mapping. However, it is possible to map outbred species as long as enough variation is present in both progenitors and loci segregation patterns can be interpreted as back cross. This approach, known as a pseudotestcross, has been widely used in plant mapping (Grattapaglia and Sederoff, 1994; Cervera et al., 2001). Although linkage maps are increasingly accessible in aquaculture [Atlantic salmon: Slettan et al. (1997); channel catfish: Waldbieser et al. (2001); rainbow trout: Sakamoto et al. (2000)], progress can be made from mapping using a pseudotestcross strategy combined with markers that do not require previous knowledge of DNA sequences (i.e., intersimple sequence repeats (ISSRS), amplified fragment length polymorphisms (AFLPs) or random amplified polymorphic DNAs (RAPDs)).

AFLP is a highly reproducible technique developed by Vos et al. (1995) where genomic DNA is digested with restriction enzymes and is ligated to adaptor sequences. Linkage maps have been developed using dominant AFLP markers in channel catfish (Liu et al., 2003), medaka (Wada et al., 1995), tilapia (Kocher et al., 1998) and zebrafish (Postlethwait et al., 1994). A drawback remains the difficulty to anchor such map to any other intra- or interspecific maps without codominant markers such as single nucleotide polymorphisms (SNPs), microsatellites or expressed sequence tags (ESTs)derived markers.

Shrimp international trade reached 950,000 tons in 1999 , being the major commodity in international trading of fish and related products (FAO, 2001). The main species under cultivation are Black Tiger Shrimp Penaeus monodon (Penaeidae; Crustacea) with a worldwide production of 450,000 tons and almost exclusively in Asia, and Penaeus (Litopenaeus) vannamei (100,000 tons) cultivated mainly in the Americas (Rosenberry, 2000). Commercial breeding programs for P. vannamei have been initiated in Colombia, USA and Ecuador, whereas Penaeus (Marsopenaeus) japonicus is being selected for increased growth in Australia. Despite its commercial importance, no successful commercial breeding programs have been reported for $P$. monodon due to problems with closure of the life cycle (Wilson et al., 2002). All the breeding programs implemented on shrimp species might benefit from the use of molecular markers and 
linkage maps. In fact two maps have been reported on shrimps (Moore et al., 1999; Wilson et al., 2002; Li et al., 2003).

In this paper, we report the development of sex specific linkage maps in $P$. vannamei using a pseudotestcross strategy and dominant AFLP markers. A family derived from a commercial stock under domestication for five generations without a known pedigree was used as mapping population. We demonstrate the feasibility of implementing molecular techniques at low cost without the need of specialized equipment. Our results show that the male and female maps of the $P$. vannamei genome had higher recombination rates than closely related species.

\section{Materials and methods}

\subsection{Biological material}

Four families were obtained from a commercial hatchery from a domesticated stock maintained for five generations. For each cross, muscle from the putative parents was stored in $99 \%$ ethanol. Animals from each cross were reared separately in 50-1 tanks from nauplii to PL12 (postlarvae) following standard protocols (Programa Iberoamericano de Ciencia y Tecnologia, 1996). From PL12 up to $1 \mathrm{~g}$, animals were grown in 500-1 indoor tanks heavily aerated with $70 \%$ weekly water exchange and at a density of one animal per liter. A $40 \%$ protein feed was fed twice a day in a volume corresponding to $5 \%$ of the calculated biomass. Growth extended for 12 weeks until the animals reached 1-g average weight. At this stage, complete animals were stored in $99 \%$ ethanol.

\subsection{AFLP analysis}

High-molecular weight genomic DNA was isolated from muscle tissue from 1-g animals and parental individuals with the CTAB method according to the protocol of Shahjahan et al. (1995). DNA samples were standardized to a concentration of $40 \mathrm{ng} / \mu \mathrm{l}$ in TE buffer and stored at $-20{ }^{\circ} \mathrm{C}$.

AFLP markers were obtained using a one-step digestion-ligation procedure as suggested by Hawthorne (2001) with some modifications. The adaptors and primers were as published by Vos et al. (1995). DNA (80 ng) was digested and ligated in the same reaction for $3 \mathrm{~h}$ at $37{ }^{\circ} \mathrm{C}$ in solution containing Tango Y buffer (MBI Fermentas) at $2 \times$ concentration, 4 pmol of EcoRI adaptor, 50 pmol of MseI adaptor, $0.5 \mathrm{mM}$ of ATP, $1 \mathrm{U}$ of ligase (Promega), 3U of Mse I (Promega), $2.5 \mathrm{U}$ of EcoRI (Promega) and MilliQ water to complete $10 \mu \mathrm{l}$ per sample. After digestion-ligation, samples were diluted 1:10 with MilliQ water. Preamplification and selective amplification were carried out with reagents from Promega. The PCR reactions contained $1 \times$ PCR buffer, $1.5 \mathrm{mM} \mathrm{MgCl}, 0.2 \mathrm{mM}$ of dNTPs and $0.008 \mathrm{U} / \mu \mathrm{l}$ of Taq polymerase. Preamplification was carried out with $2 \mu \mathrm{l}$ of the ligated product and $0.2 \mu \mathrm{M}$ of both EcoRI and $\mathrm{Mse}$ I preselective primers in a total volume of $20 \mu \mathrm{l}$. Selective amplification was accomplished with $0.6 \mu \mathrm{M}$ of selective forward and reverse primers using $1.2 \mu \mathrm{l}$ of a 1:10 dilution of preamplified product in a 12 $\mu \mathrm{l}$ total volume. PCR conditions were as follows: initial denaturation at $94{ }^{\circ} \mathrm{C}$ for $2 \mathrm{~min}$; 
35 cycles at $94{ }^{\circ} \mathrm{C}$ for $30 \mathrm{~s}, 54{ }^{\circ} \mathrm{C}$ for $60 \mathrm{~s}$ and $72{ }^{\circ} \mathrm{C}$ for $60 \mathrm{~s}$ and a final extension for $60 \mathrm{~s}$ at $72{ }^{\circ} \mathrm{C}$.

PCR products (12 $\mu \mathrm{l}$ per sample) were separated by vertical electrophoresis in 1-mmthick nondenaturating gels containing 8\% acrylamide/bisacrylamide mixture (29:1). For DNA visualization, a silver staining protocol with some modification was used (Dinesh et al., 1995). In short, gels were exposed to a fix solution (10\% ethanol and 5\% acetic acid) for $15 \mathrm{~min}$, silver impregnated for $15 \mathrm{~min}$ (11 $\mathrm{mM}$ of silver nitrate), washed for $1 \mathrm{~min}$ (deionized water), developed for 10 to $15 \mathrm{~min}(0.75 \mathrm{M} \mathrm{NaOH}$ and $85 \mathrm{mM}$ formaldehyde) and the reaction was stopped for $5 \mathrm{~min}$ ( $70 \mathrm{mM}$ sodium carbonate). Solutions were reused up to eight times the same day. Gels were air-dried for $1 \mathrm{~h}$ and wrapped in cellulose sheets to avoid gel breakage. Visual scoring was carried out on clearly visible and well-defined bands. Band names were assigned according to the presence or absence in the female parental phenotype as fe\# or ma\# where \# represents the order of appearance of the segregating band during the scoring. Genotypes were saved in an Excel worksheet. Gels were photographed using the Kodak DC120 system with a light box for documentation purposes.

\subsection{Linkage analysis}

A Chi-square test for the 1:1 or 3:1 segregation ratios was applied to each locus. Loci with $P$ values lower than 0.05 in both tests were discarded from further analysis. Data were exported to the Map Manager QTX software (Manly et al., 2001) and a set of framework linkage groups was determined with a minimum LOD score of 3.0. Nonlinked markers were assigned to the framework linkage groups with a LOD value of 2.0 using the Distribute command. Because assignment by Distribute is dependent on knowledge of phase and the coupling/repulsion phase is not known for dominant marker data, remaining nonlinked markers were phase-changed with the Flip command and assigned to the framework set with a LOD score of 2.0 using the Distribute command. Each linkage group was inspected using the built-in map and statistics windows included in the Map Manager QTX software. Genetic distances (cM) were calculated using the Kosambi function. In order to avoid map expansion, double recombinants were checked. Genotypes were excluded from the specific linkage group analysis if double crossovers were detected at a distance lower than $20 \mathrm{cM}$. Linkage groups generated with Map Manager were checked with the Mapmaker/EXP 3.0 software (Lander et al., 1987) using the Group, Compare and Ripple commands with the error detection option on.

The genome size was estimated using the methods 3 and 4 as described by Chakravarti et al. (1991). The markers linked with LOD scores between 3.0 and 9.0 were used for both calculations to avoid overestimation of genome size due to tightly linked markers. The marker distribution was considered using a normality test (Kolmogorov-Smirnoff test) on standardized distances between adjacent markers. Similarly, the Pearson's Correlation between the number of markers per linkage group and the total length of the linkage group were calculated in order to evaluate the random distribution of markers throughout the maps. Drawing of a histogram, the normality test and the Pearson's Correlation were calculated with the Statistica software version 6.0 (Statsoft). Linkage groups were drawn with the Mapinspect software version 1.0 (http://www.dpw.wau.nl/pv/pub/). 


\section{Results}

\subsection{Parentage testing}

The DNA from both parents and 10 siblings of each family were used for parentage analysis by inspection of shared bands between the putative progenitor and the progeny using eight AFLP primer combinations. Fingerprints from both putative male and female parents from two families gave profiles that were incompatible with the progeny being tested. In two other families, both males also gave unexpected fingerprints. These results left us with the two families where only the female parent was known. The female and 42 progenies from one of these families were used as the mapping population reported in this study.

\subsection{Map construction}

A total of 103 primer combinations were used for the map construction, which gave 741 segregating bands (Table 1). The average number of bands per gel was 54 while the average number of polymorphic bands scored was 7.1 per gel.

Assignment of paternal and maternal bands was based on a sequential process. First, all markers were tested with a Chi-square test for $1: 1$ or $3: 1$ segregation pattern at the $P=0.05$ level. About $64 \%$ of the segregating markers fitted the $1: 1$ model, whereas $24.5 \%$ fitted the 3:1 model. Bands with the 1:1 segregation were assigned as maternal if they were present on the maternal fingerprint and paternal if they were not. A total of 249 bands were assigned to the maternal and 228 to paternal parents respectively. For the remaining bands, 181 fitted the 3:1 model while 62 fitted both, the 1:1 and the 3:1 models. Twenty-one bands fitted neither segregation pattern.

Using the pseudotestcross strategy (Grattapaglia and Sederoff, 1994), which assumes that a 1:1 ratio implies one parent is heterozygous and the other is homozygous for the band absent, two maps were obtained. The female map comprised 212 markers which formed 51 linkage groups covering a total length of $2771 \mathrm{cM}$ (Fig. 1). One linkage group of two markers with a distance of $0 \mathrm{cM}$ was excluded from Fig. 1. A total of 155 and 57 markers were grouped with LOD scores higher than 3.0 or between 2.0 and 3.0, respectively. Total of $86 \%$ of the maternally derived markers was placed on the female map. Marker numbers per linkage group ranged from 2 to 10, with an average of 4.2 markers. Total linkage group length varied from 0 to $188 \mathrm{cM}$, with an average of $55.3 \mathrm{cM}$. Average distance between markers was $17.1 \mathrm{cM}$ and ranged from 0 to $30 \mathrm{cM}$.

In the male map, 182 markers were grouped to 47 linkage groups covering a total distance of $2116 \mathrm{cM}$ (Fig. 2). The initial framework map comprised 135 markers grouped with the LOD score higher than 3.0 and 47 markers were added in with the LOD score between 2.0 and 3.0. The markers ma342 and ma410 were grouped together with the LOD value of 2.0 as a single linkage group. Eighty percent of the paternal markers were mapped on the male map. The linkage group length varied from 2 to $159 \mathrm{cM}$, with an average of 45 $\mathrm{cM}$. Number of markers per linkage group varied from 2 to 11 with an average of 3.9. The distance between markers ranged from 2 to $30 \mathrm{cM}$ with an average spacing of $15.6 \mathrm{cM}$. The comparison of the linkage associations and marker order between the software 
packages Map Manager and Mapmaker gave the same results. However, Map Manager allowed for easier visualization of linkage statistics, genotypes and maps.

\subsection{Map characteristics}

The estimated genome size varied according to the calculation methods. The genome size of the female was $5407 \mathrm{cM}$, and $4333 \mathrm{cM}$ for the male genome, using method 3 as described by Chakravarti et al. (1991). Method 4 from the same authors gave an estimate of 4445 and $3584 \mathrm{cM}$ for the female and male genome, respectively. Considering the more conservative estimates derived from method 4 , the genome coverage of our map reached $62 \%$ for the female and $59 \%$ for the male genome.

The normality tests on linkage intervals for the male and female data showed a nonnormal distribution. Visual inspection of the histograms (Fig. 3) displayed clustering of standardized genetic distances on the right and left side of the distribution for the male and female maps. In addition, the Pearson's Correlation Coefficient between linkage group length and number of markers per linkage group was high (0.83 and 0.79) and highly significant $(P<0.001)$ for both groups of data. This shows that the AFLP markers on our map were randomly distributed. However, some cases were seen where markers amplified with the same primer combination mapped to the same linkage group, i.e., FE-1. During data collection, we found various markers on the same gel that segregated with an identical pattern and which were not included in our analysis.

\section{Discussion}

We have developed sex-specific maps of the white shrimp P. vannamei by using a commercial family derived from a cross with genotypic information from one progenitor. The implemented protocols allowed for high data throughput at low cost. The use of silver staining for visualization of DNA fragments gave a high detection ratio (54 bands per gel) which compares favorably with other projects using radioactive and fluorescent automated detection (50 to 75 bands per primer pair) (Wilson et al., 2002). Silver staining is a technique easily implemented and that does not require the use of specialized equipment such as automated sequencers. In the course of our work, we processed a maximum of 8 gels per day with the same staining solutions. However, it might be possible to develop even more gels using the same solutions, which might reduce the costs even more. Additionally, the silver staining protocol we used works at room temperature without cooling of the solutions as in the popular protocol developed by Bassam et al. (1991).

Microsatellite genotyping is very useful for parentage assignment in shrimp (Moore et al., 1999; Wilson et al., 2002). With dominant markers, such as AFLPs, each DNA band from a parent should be present in either $100 \%$ or $50 \%$ of the progeny depending on the parent being homozygous or heterozygous. In a progeny sample comprising 10 animals, the probability of absence of a 1:1 ratio parental segregating band is 0.0015 for a Chisquare distribution. Similarly, with the assumption of independence between most of the segregating bands amplified with a primer pair, probability of absence of various AFLP 
Table 1

Number of polymorphic bands (open numbers) and corresponding coding of the segregating bands (in brackets) generated with 103 Eco +3 and Mse +3 AFLP primer combinations in the P. vannamei mapping panel

\begin{tabular}{|c|c|c|c|c|c|c|c|c|c|}
\hline \multicolumn{10}{|l|}{ MSE } \\
\hline $\mathrm{ECO}$ & CAA & $\mathrm{CAC}$ & $\mathrm{CAG}$ & CAT & CGA & CGC & CGG & CGT & TOTAL \\
\hline AAA & $1(1)$ & 13 (87-99) & $8(203-210)$ & $4(289-292)$ & $4(369-372)$ & $8(456-463)$ & $15(550-564)$ & $6(671-676)$ & 58 \\
\hline $\mathrm{AAC}$ & $9(2-10)$ & $7(100-106)$ & $7(211-217)$ & $13(293-305)$ & $3(373-375)$ & $11(464-474)$ & $14(565-578)$ & $1(677-677)$ & 56 \\
\hline AAG & $5(11-15)$ & $4(107-110)$ & $5(218-222)$ & $6(306-311)$ & $3(376-378)$ & $13(475-487)$ & $3(579-581)$ & $8(678-685)$ & 42 \\
\hline AAT & $8(16-23)$ & $20(111-130)$ & $8(223-230)$ & $10(312-321)$ & $7(379-385)$ & $6(488-493)$ & $13(582-594)$ & $4(686-689)$ & 68 \\
\hline ACA & $16(24-39)$ & $6(131-136)$ & 0 & $3(322-324)$ & $5(386-390)$ & $4(494-497)$ & $10(595-604)$ & $9(690-698)$ & 37 \\
\hline $\mathrm{ACC}$ & $7(40-46)$ & $10(137-146)$ & $9(231-239)$ & $6(325-330)$ & $9(391-399)$ & $7(498-504)$ & $10(605-614)$ & $9(699-707)$ & 60 \\
\hline $\mathrm{ACT}$ & 0 & $3(156-158)$ & $2(245-246)$ & $8(336-343)$ & $9(415-423)$ & $1(515-515)$ & $7(621-627)$ & $5(713-717)$ & 35 \\
\hline AGA & $12(55-66)$ & $6(159-164)$ & $11(247-257)$ & $8(344-351)$ & $6(424-429)$ & $11(516-526)$ & $2(628-629)$ & 0 & 44 \\
\hline AGC & 0 & $7(165-171)$ & $13(258-270)$ & $7(352-358)$ & $14(430-443)$ & $11(527-537)$ & $14(630-643)$ & $1(718-718)$ & 67 \\
\hline AGG & $8(67-74)$ & $12(172-183)$ & $5(271-275)$ & $2(359-360)$ & $5(444-448)$ & $1(538-538)$ & $9(644-652)$ & $11(719-729)$ & 45 \\
\hline AGT & $4(75-78)$ & $6(184-189)$ & $8(276-283)$ & 0 & $3(449-451)$ & $4(539-542)$ & $14(653-666)$ & $12(730-741)$ & 47 \\
\hline \multirow[t]{2}{*}{ ATA } & $8(79-86)$ & $13(190-202)$ & $5(284-288)$ & $8(361-368)$ & $4(452-455)$ & $7(543-549)$ & $4(667-670)$ & $\mathrm{NE}$ & 41 \\
\hline & 86 & 116 & 86 & 80 & 87 & 94 & 121 & 71 & 741 \\
\hline
\end{tabular}

$\mathrm{NE}=$ not evaluated. 


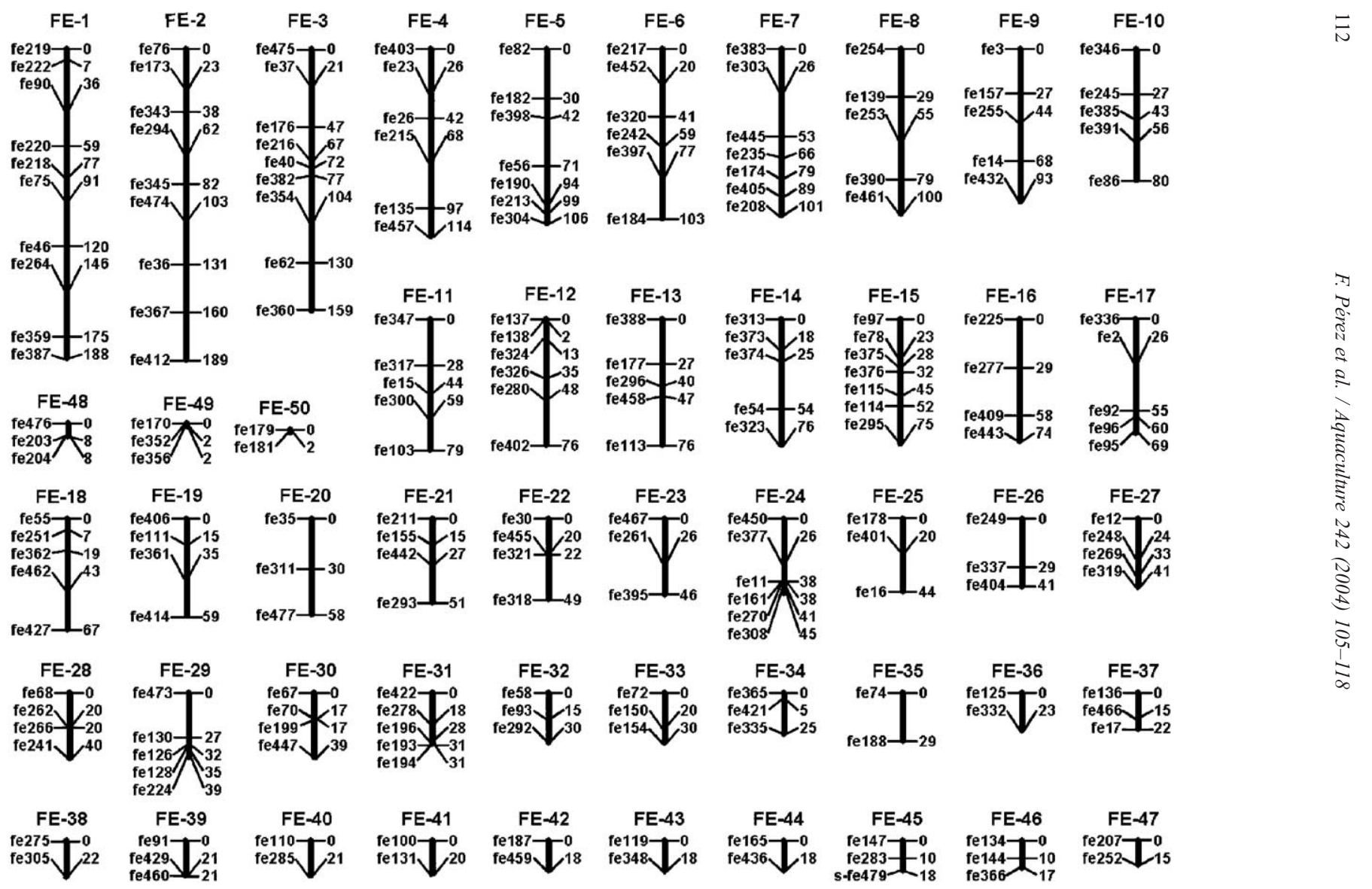

Fig. 1. Female map of $P$. vannamei based on AFLP markers. Female markers match to the segregating markers present in the female progenitor. Linkage distance was in Kosambi units (cM). Marker numbers correspond to the order of appearance during the scoring process. 

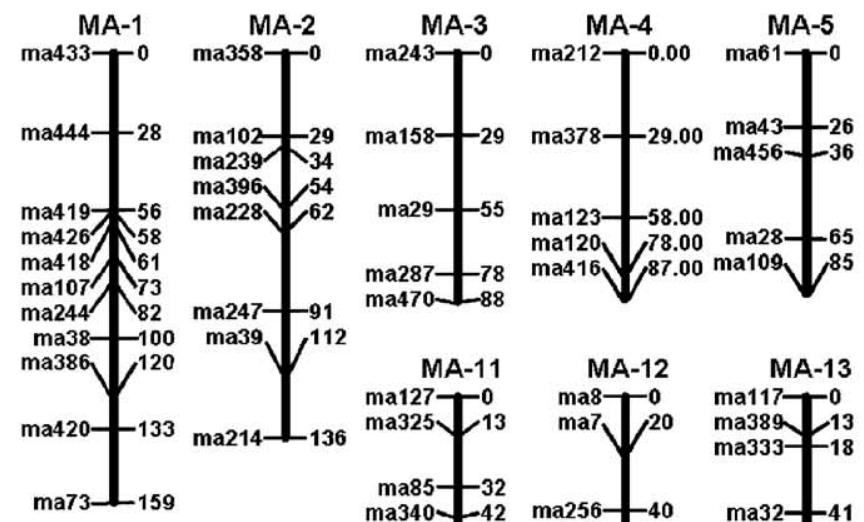

$\operatorname{ma} 287-78$
$\operatorname{ma470}-88$ $\operatorname{ma28}-65$
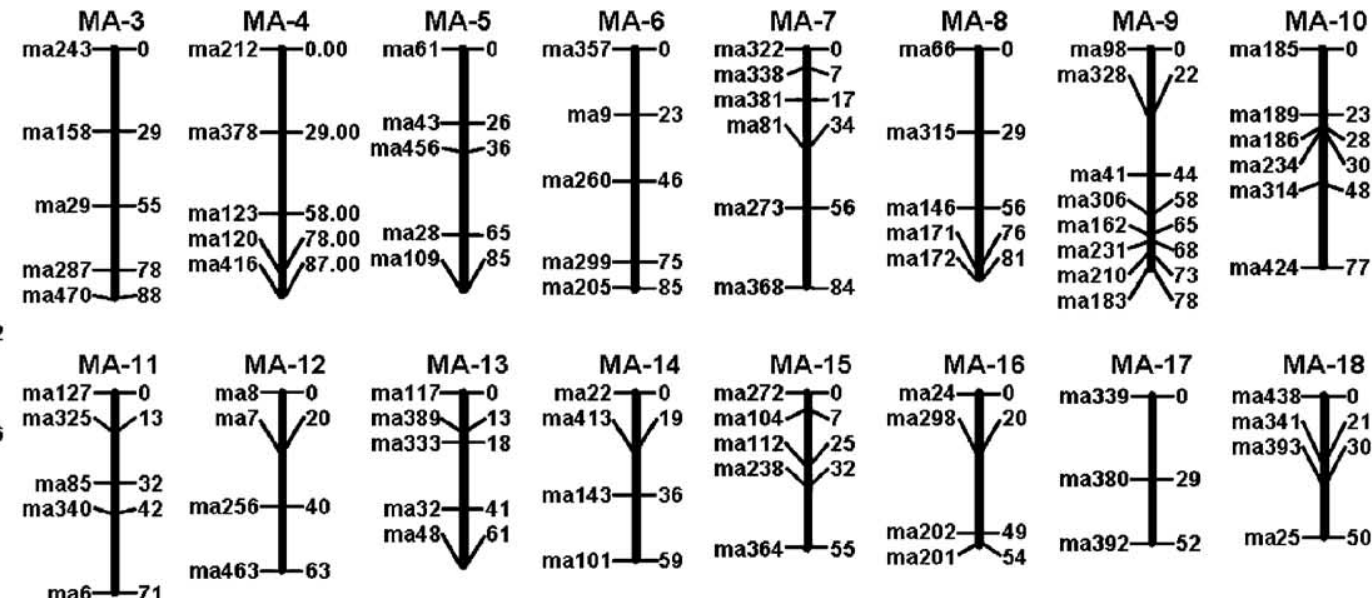

MA-14

MA-14 MA-15 ma22-0 ma272-0 ma272 T7 ${ }_{\text {ma202 }}^{\text {Ma24-16 }}$

MA-17 ma339 $\longrightarrow 0$ ma143-36 ma238 32

ma380-29 ma364- -55 ma101-L9
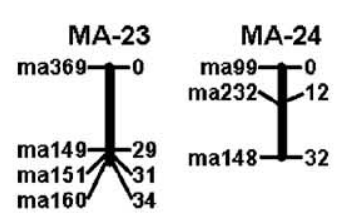

MA-24 ma99-0 ma232 -12 ma148- - 2 ma71 -29 MA-22
ma227
ma274-26 -36 ma133-24 $\operatorname{ma289-49} \operatorname{ma141}_{\operatorname{ma284}-41}$ MA-21
ma180-25
ma250
ma88
ma468
ma175 $\operatorname{ma160}_{34}$

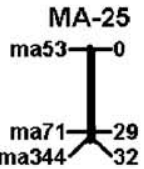

MA-26 ma168-0 ma4- -29 ma392- -52

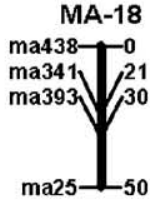

$\underset{\operatorname{ma132}}{\operatorname{ma}}-\frac{-29}{31}$


$\stackrel{\text { MA-35 }}{\operatorname{ma21}_{\text {ma439 }}^{\text {M }}}$

MA-36 ma129-0 ma316 L2

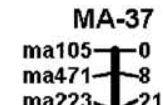

MA-38

ma351-0 ma384 ${ }^{21}$



ma153
MA-47

ma65-1 0 ma60 0 ma118-0 ma435-0 ma42-0

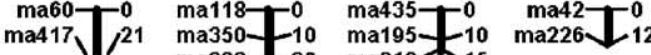
ma236 5 ma431 ב-8

Fig. 2. Male map of P. vannamei based on AFLP markers. Male markers corresponded to the segregating markers not present in the female progenitor. Linkage distance 
markers segregating in a 1:1 ratio is multiplicative. If the putative parent shows two bands that are not present in the progeny, the probability of getting such sample is 0.0000025 . Additionally, the presence of bands in the progeny which are absent from a putative progenitor complement those results. This situation corresponds to a simple pedigree analysis that can be solved by the exclusion principle (Danzmann, 1997). In our work, we found two situations: absence of major bands from a putative progenitor in the tested progeny and presence of major bands in all the progeny that were not present in the putative progenitor tested. Only two females from the eight evaluated individuals showed all their bands to be present in the four evaluated families.
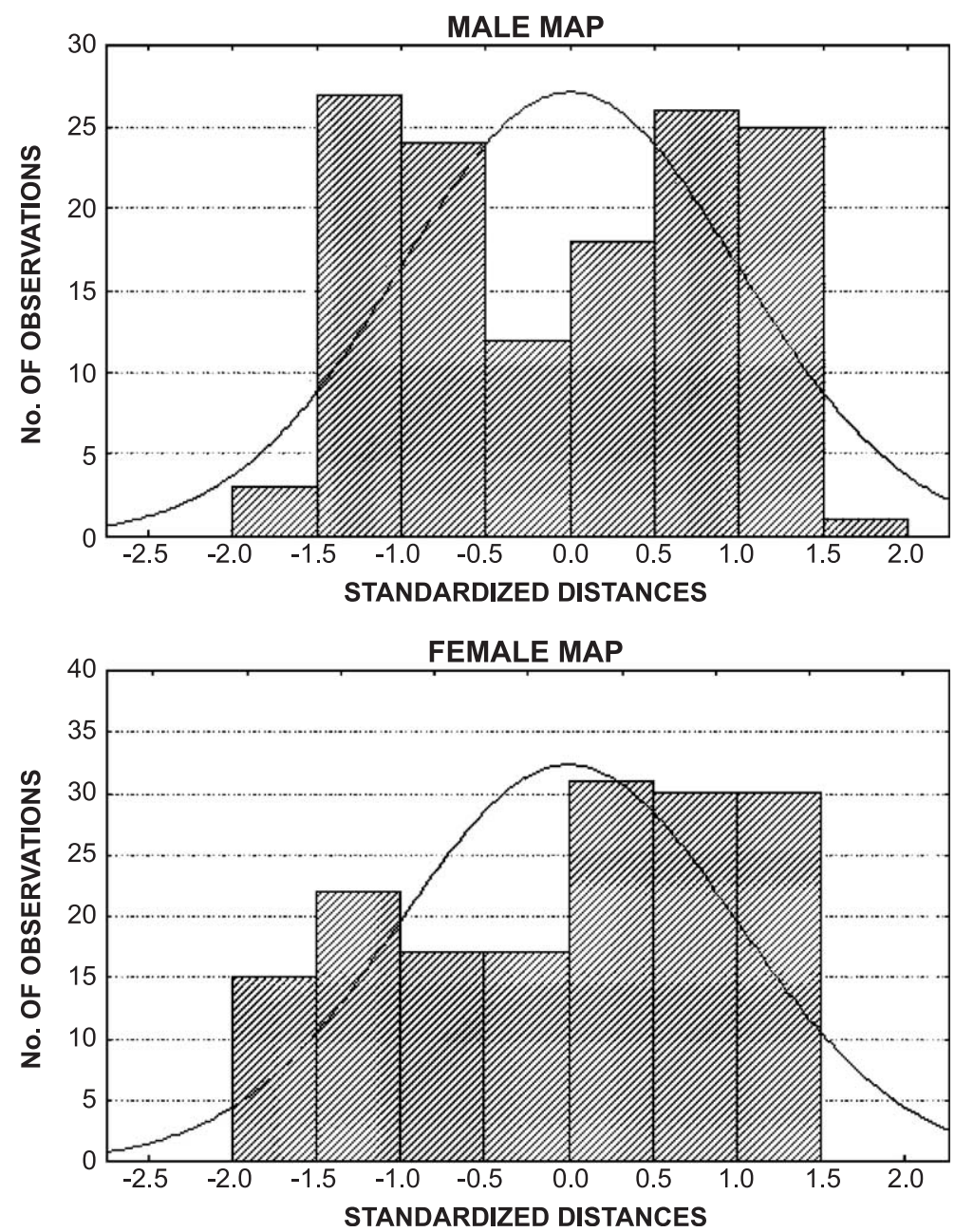

Fig. 3. Distribution of normalized Kosambi distances on the male and female maps of P. vannamei. Both distributions are nonnormal according to the Kolgomorov-Smirnov test and show clustering on the left- and righthand sides. 
The number of polymorphic loci detected ( 7.1 bands per primer combination) is lower than the 14.2 and 10.0 loci reported in P. japonicus and P. monodon (Moore et al., 1999; Li et al., 2003; Wilson et al., 2002). It is not clear if this difference is due to intrinsic characteristics of the $P$. vannamei genome or a high level of inbreeding in our mapping population.

In $P$. monodon, the percentage of markers segregating in a 1:1 (backcross) and in a 3:1 (intercross) models was $72.3 \%$ and 27.7\%, respectively (Wilson et al., 2002). Those proportions in our data, at $64 \%$ and $24.5 \%$, respectively, are similar. The difference may be due to the use of the Chi-square test based on the genotypic information derived from only one parent and small sample size, which gave $8.4 \%$ of bands fitting both models. Similar to the data reported in P. japonicus (Li et al., 2003), we found $2.8 \%$ of the scored bands fitting neither segregation model at the $P=0.01$ level. This figure could be higher because we did not score loci that had too many or too few bands and that were initially considered as artifacts during the scoring process. Segregation distortion has been reported in plants (Marques et al., 1998) and animals (Li and Guo, 2004; Liu et al., 2003; Tan et al., 2001) and may be caused by differential survival due to the presence of lethal alleles or sampling errors on small groups (Causse et al., 1994). In P. stylirostris, heterozygous genotypes were preferentially found in inbred populations (Bierne et al., 2000) probably due to the covering of lethal genes, which may explain our distorted segregation. However, it is arguable that the very small population size (42 animals) was the main reason for segregation distortion in this study.

Mapping strategies normally rely on information from both parents and specific pedigrees. However, linkage analysis is possible in species where meiosis suppression occurs in one sex, such as is the case with the silkworm (Tan et al., 2001). An analogous situation arises when dealing with an interspecific mapping population in plants (Cervera et al., 2001). In our study, the maternal parental genotypic data were used as the references to deduce the information from the male progenitor. The assignation of maternal and paternal markers was similar for both the male and female groups $(47.8 \%$ and $52.2 \%$, respectively).

The P. vannamei haploid genome has 44 chromosomes (Ramos, 1997). Our female and male maps comprise 51 and 47 linkage groups, respectively. Presence of extra linkage groups can be explained by the lack of intermediate markers between linkage groups belonging to the same chromosome. Low genome coverage could account also for the nonlinked markers observed in our study.

In a saturated map, the frequency of genetic distances with markers randomly located should show a normal distribution (Krutovskii et al., 1998). Clustering due to recombination suppression, a phenomenon occurring mainly near to the centromeric areas should be located on the left side of the distribution where short distances are to be found. On the other hand, a lowly saturated map might show clustering on the right side of the distribution because big gaps did not have enough markers. The distribution of markers in our male and female maps showed a nonnormal distribution with clustering on both sides.

When markers are randomly distributed throughout the genome, the correlation between number of markers per linkage group and length of the linkage groups must be 
linear because the bigger the chromosome, the higher number of markers than can be grouped (Cervera et al., 2001). Correlation analysis in our data showed a highly significant relationship between linkage group length and number of markers. We conclude that the AFLP markers in P. vannamei are randomly distributed among different chromosomes for both the male and female maps.

The genome size estimate for P. japonicus was $2300 \mathrm{cM}$ (Moore et al., 1999), whereas our effective coverage was $2771 \mathrm{cM}$ for the female and $2116 \mathrm{cM}$ for the male maps of $P$. vannamei. The estimation of genome size with our data showed higher figures for both maps (4445 and $3583 \mathrm{cM}$ with the more conservative estimates). Nevertheless, the estimate in $M$. japonicus was made using a different technique (bootstrapping) that might give different results with low genome coverage. However, Li et al. (2003) report a second-stage map with increased coverage in M. japonicus, with a total length of $1780 \mathrm{cM}$ for the male map that is close to the genome size estimate given by Moore et al. (1999). In $P$. vannamei, the clustering on the right side of distribution of markers in both maps, extra linkage groups and the estimated genome size indicate that our maps have a low-density coverage. Our total map length for both the male and female maps, added to the lowdensity coverage shows evidence that the recombination rates in P. vannamei are higher than closely related species.

In $P$. vannamei, the female map was $24 \%$ larger than the male map. Similarly, the genome size calculations show that the recombination rate might be higher in females than in males. Wilson et al. (2002) found a similar trend in P. monodon with a higher recombination rate in the female genome. The mean recombination rate is sex-dependent in humans, with higher recombination rates in the female than in the male (Lynn et al., 2000; Matise et al., 2002). Zebrafish and rainbow trout also show recombination suppression in the males (Singer et al., 2002; Sakamoto et al., 2000). Differences in meiotic behavior can be used to speed up positional cloning and isolation of markers tightly linked to desirable traits.

Integration of the male and female maps was not possible using the markers segregating in 3:1 ratio, which are equivalent to an intercross configuration. With dominant markers, the intercross configuration gives information only in one-quarters of the population, which corresponds to the homozygous recessive genotypes without bands. In our study some intercross loci could be placed in linkage groups but the order support was low and in most cases the 1:1 marker order was changed. In order to integrate both maps, codominant markers should be used.

The AFLP approach used in this study was effective to generate linkage information in $P$. vannamei, a species with scarce genetic information available. High genome coverage is necessary for QTL mapping and marker-assisted selection. Codominant markers, including ESTs, microsatellites, RFLPs and sequence-characterized amplified regions (SCARs) should be placed on this map to improve its usefulness as a breeding and genetic tool (see also Alcivar-Warren, 2001). Full genome coverage as needed for QTL mapping, requires markers linked at a maximum distance of $20 \mathrm{cM}$. Due to the clustering observed in our map and the calculated genome size, over 300 codominant loci should be mapped. Alternatively, a combined strategy using dominant markers and at least one codominant marker anchored to each chromosome might be possible. 


\section{Acknowledgements}

This work was supported by the Ecuadorian Science and Technology Foundation (FUNDACYT) under the BID-210 Project, the Belgian Technical Cooperation (BTC) and the Interuniversitary Flemish Cooperation (VLIR). We thank M. Maldonado for support in raising the experimental animals and L. Tomala for help with laboratory work.

\section{References}

Alcivar-Warren, A., 2001. ShrimpMap: a genetic approach to understand immune response and disease resistance in shrimp. Aquaculture 2001: Book of Abstracts. World Aquaculture Society, Baton Rouge LA, USA, pp. 11. Abstract only.

Bassam, B.J., Caetano-Anolles, G., Gresshoff, P.M., 1991. Fast and sensitive silver staining of DNA in polyacrylamide gels. Anal. Biochem. 196, 80-83.

Bierne, N., Bezuart, I., Vonau, V., Bonhomme, F., Bedier, E., 2000. Microsatellite-associated heterosis in hatchery-propagated stocks of the shrimp Penaeus stylirostris. Aquaculture 184, 203-219.

Causse, M.A., Fulton, T.M., Cho, Y.G., Ahn, S.N., Chunwongse, J., Wu, K.S., Xiao, J.H., Yu, Z.H., Ronald, P.C., Harrington, S.E., Second, G., Mccouch, S.R., Tanksley, S.D., 1994. Saturated molecular map of the rice genome based on an interspecific backcross population. Genetics 138, 1251-1274.

Cervera, M.T., Storme, V., Ivens, B., Gusmao, J., Liu, B.H., Hostyn, V., Van Slycken, J., Van Montagu, M., Boerjan, W., 2001. Dense genetic linkage maps of three Populus species (Populus deltoides, P. nigra and P. trichocarpa) based on AFLP and microsatellite markers. Genetics 158, 787-809.

Chakravarti, A., Lasher, L.K., Reefer, J.E., 1991. A maximum-likelihood method for estimating genome length using genetic-linkage data. Genetics 128, 175-182.

Danzmann, R.G., 1997. PROBMAX: a computer program for assigning unknown parentage in pedigree analysis from known genotypic pools of parents and progeny. J. Heredity 88, 333-337.

Dinesh, K.R., Chan, W.K., Lim, T.M., Phang, V.P.E., 1995. RAPD markers in fishes - an evaluation of resolution and reproducibility. Asia-Pac. J. Molec. Biol. Biotechnol. 3, 112-118.

Food and Agriculture Organization FAO, 2001. Fish and fisheries products. Food outlook 1, February 2001. FAO/ GIEWS 11, 29.

Grattapaglia, D., Sederoff, R., 1994. Genetic linkage maps of Eucalyptus grandis and Eucalyptus urophylla using a pseudo-testcross: mapping strategy and RAPD markers. Genetics 137, 1121-1137.

Hawthorne, D.J., 2001. AFLP-based genetic linkage map of the Colorado potato beetle Leptinotarsa decemlineata: sex chromosomes and a pyrethroid-resistance candidate gene. Genetics 158, 695-700.

Kocher, T.D., Lee, W.J., Sobolewska, H., Penman, D., McAndrew, B., 1998. A genetic linkage map of a cichlid fish, the tilapia (Oreochromis niloticus). Genetics 148, 1225-1232.

Krutovskii, K., Vollmer, S., Sorensen, F., Adams, W., Knapp, S., Strauss, S., 1998. RAPD genome maps of Douglas-Fir. J. Heredity 89 (3), 197-205.

Lander, E.S., Green, P., Abrahamson, J., Barlow, A., Daly, M.J., Lincoln, S.J., Newburg, L., 1987. MAPMAKER: an interactive computer package for constructing primary genetic linkage maps of experimental and natural populations. Genomics 1, 174-181.

Li, L., Guo, X., 2004. AFLP-based genetic linkage maps of the pacific oyster Crassostrea gigas Thunberg. Mar. Biotechnol. 6, 26-36.

Li, Y., Byrne, K., Miggiano, E., Whan, V., Moore, S., Keys, S., Crocos, P., Preston, N., Lehnert, S., 2003. Genetic mapping of the kuruma prawn Penaeus japonicus using AFLP markers. Aquaculture 219, 143-156.

Liu, Z., Karsi, A., Li, P., Cao, D., Dunham, R., 2003. An AFLP-based genetic linkage map of channel catfish (Ictalurus punctatus) constructed by using an interspecific hybrid resource family. Genetics 165, 687-694.

Lynn, A., Kashuk, C., Petersen, M.B., Bailey, J.A., Cox, D.R., Antonarakis, S.E., Chakravarti, A., 2000. Patterns of meiotic recombination on the long arm of human chromosome 21. Genome Res. 109, 1319-1332.

Manly, K.F., Cudmore, R.H., Meer, J.M., 2001. Map manager QTX, cross-platform software for genetic mapping. Mamm. Genome 12, 930-932. 
Marques, C.M., Araujo, J.A., Ferreira, J.G., Whetten, R., O’malley, D.M., Liu, B.H., Sederoff, R., 1998. AFLP genetic maps of Eucalyptus globulus and E. tereticornis. Theor. Appl. Genet. 96, 727-737.

Matise, T.C., Porter, C.J., Buyske, S., Cuttichia, A.J., Sulman, E.P., White, P.S., 2002. Systematic evaluation of map quality: human chromosome 22. Am. J. Hum. Genet. 70, 1398-1410.

Moore, S.S., Whan, V., Davis, G.P., Byrne, K., Hetzel, D.J.S., Preston, N., 1999. The development and application of genetic markers for the kuruma prawn Penaeus japonicus. Aquaculture 173, 19-32.

Postlethwait, J.H., Johnson, S.L., Midson, C.N., Talbot, W.S., Gates, M., Ballinger, E.W., Africa, D., Andrews, R., Carl, T., Eisen, J.S., et al., 1994. A genetic linkage map for the zebrafish. Science 264, 699-703.

Programa Iberoamericano de Ciencia y Tecnologia para el Desarrollo Subprograma II Acuicultura, 1996. Producao de Pós-larvas de Camarao Marinho. Curso internacional, Florianópolis, Brasil.

Ramos, R.C., 1997. Chromosome studies on the marine shrimps Penaeus vannamei and P. californiensis (Decapoda). J. Crustac. Biol. 17, 666-673.

Rosenberry, R., 2000. World shrimp farming 2000. Shrimp News Int., 6-103.

Sakamoto, T., Danzmann, R.G., Gharbi, K., Howard, P., Ozaki, A., Khoo, S.K., Woram, R.A., Okamoto, N., Ferguson, M.M., Holm, L.E., Guyomard, R., Hoyheim, B., 2000. A microsatellite linkage map of rainbow trout (Oncorhynchus mykiss) characterized by large sex-specific differences in recombination rates. Genetics $155,1331-1345$.

Savelkoul, P.H., Aarts, H.J., de Haas, J., Dijkshoorn, L., Duim, B., Otsen, M., Rademaker, J.L., Schouls, L., Lenstra, J.A., 1999. Amplified-fragment length polymorphism analysis: the state of an art. J. Clin. Microbiol. 37, 3083-3091.

Shahjahan, R.M., Roger, K.J.H., Leopold, R.A., Devault, J.D., 1995. Lower incubation-temperature increases yield of insect genomic DNA isolated by the CTAB method. BioTechniques 19, 332-334.

Singer, A., Perlman, H., Yan, Y.L., Walker, C., Corley-Smith, G., Brandhorst, B., Postlethwait, J., 2002. Sexspecific recombination rates in zebrafish (Danio rerio). Genetics 160, 649-657.

Slettan, A., Olsaker, I., Lie, O., 1997. Segregation studies and linkage analysis of Atlantic salmon microsatellites using haploid genetics. Heredity 78, 620-627.

Smith, J., Paton, I.R., Murray, F., Crooijmans, R.P., Groenen, M.A., Burt, D.W., 2002. Comparative mapping of human chromosome 19 with the chicken shows conserved synteny and gives an insight into chromosomal evolution. Mamm. Genome 13, 310-315.

Tan, Y.D., Wan, C., Zhu, Y., Lu, C., Xiang, Z., Deng, H.W., 2001. An amplified fragment length polymorphism map of the silkworm. Genetics 157, 1277-1284.

Vos, P., Hogers, R., Bleeker, M., Reijans, M., Lee, Th. van der, Hornes, M., Frijters, A., Pot, J., Peleman, J., Kuiper, M., Zabeau, M., 1995. AFLP: a new technique for DNA fingerprinting. Nucleic Acids Res. 23, 4407-4414.

Wada, H., Naruse, K., Shimada, A., Shima, A., 1995. Genetic linkage map of a fish, the Japanese medaka Oryzias latipes. Mol. Mar. Biol. Biotechnol. 4, 269-274.

Waldbieser, G.C., Bosworth, B.G., Nonneman, D.J., Wolters, W.R., 2001. A microsatellite-based genetic linkage map for channel catfish Ictalurus punctatus. Genetics 158, 727-734.

Wilson, K., Li, Y., Whan, V., Lehnert, S., Byrne, K., Moore, S., Pongsomboon, S., Tassanakajon, A., Rosenberg, G., Ballment, E., Fayazi, Z., Swan, J., Kenway, M., Benzie, J., 2002. Genetic mapping of the black tiger shrimp Penaeus monodon with amplified fragment length polymorphism. Aquaculture 204, 297-309. 